\title{
Central Proteins of Plasma in Response to Low- Level Laser Therapy Involve in Body Hemostasis and Wound Repair
}

\author{
Babak Arjmand $^{1}{ }^{\circledR}$, Reza Vafaee ${ }^{2}$, Mohhamadreza Razzaghi ${ }^{3}$, Mostafa Rezaei Tavirani $^{*{ }^{\circledR}}$, Alireza \\ Ahmadzadeh$^{2}$, Sina Rezaei Tavirani ${ }^{5}$, Mostafa Hamdieh ${ }^{6}$
}

${ }^{1}$ Cell Therapy and Regenerative Medicine Research Center, Endocrinology and Metabolism Molecular-Cellular Sciences Institute, Tehran University of Medical Sciences, Tehran, Iran ${ }^{2}$ Proteomics Research Center, Shahid Beheshti University of Medical Sciences, Tehran, Iran ${ }^{3}$ Laser Application in Medical Sciences Research Center, Shahid Beheshti University of Medical Sciences, Tehran, Iran ${ }^{4}$ Proteomics Research Center, Faculty of Paramedical Sciences, Shahid Beheshti University of Medical Sciences, Tehran, Iran

${ }^{5}$ Gastroenterology and Liver Diseases Research Center, Research Institute for Gastroenterology and Liver Diseases, Shahid Beheshti University of Medical Sciences, Tehran, Iran

${ }^{6}$ Department of Psychosomatic, Taleghani Hospital, Faculty of Medicine, Shahid Beheshti University of Medical Sciences, Tehran, Iran

\section{*Correspondence to Mostafa Rezaei Tavirani, Proteomics Research Center, Faculty of Paramedical Sciences, Shahid Beheshti University of Medical Sciences, Tehran, Iran. Email: tavirany@yahoo.com \\ Published online December 30, 2020

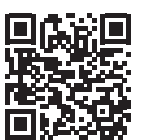

\section{Introduction}

Low-level laser therapy (LLLT) as an efficient method is applied in various fields of medicine. Wound healing, dentistry, neurorehabilitation, and several different fields are interested to apply LLLT for therapeutic purposes. ${ }^{1-3}$ Better understanding and promotion of LLLT efficacy implies that the molecular mechanism of the affected parts of the body treated via LLLT should be explored., ${ }^{4,5}$ Proteomics as a high-throughput method is a useful tool to study protein expression change due to intervention in the patient condition. Results of a proteomic experience are a set of proteins that are dysregulated after intervention in the biological samples. Therefore, many diseases are studied via proteomics to determine the dysregulated protein production processes in the body. ${ }^{6,7}$

Bioinformatics is the other tool that is applied in medicine to solve the complex nature of high-throughput findings. Since the results of high-throughput experiences need further analysis to be simplified, bioinformatics provides new concepts via complex analysis. ${ }^{8,9}$ There are various approaches in bioinformatics that are applied to analyze biological systems including diseases. Network analysis, gene ontology, artificial neural networks, and many other tools which are related to bioinformatics are applied to analyze the complex information in medicine and to present the new concepts. ${ }^{10-12}$

Protein-protein interaction (PPI) network analysis studies the relationship between large numbers of proteins or genes. In this approach, the studied proteins are linked to construct a network. The elements of the network are connected to the neighbors in different patterns. Based on the distribution of the connections between the nodes of the network, two kinds of networks, namely the scale-free and non-scale-free networks, are introduced. Generally, PPI networks of the large numbers of connected proteins are scale-free. In such a 
network, there are a few numbers of nodes (as central nodes) which are discriminated from the others by the differentiated numbers of connections. ${ }^{13,14}$ PPI network analysis is applied in medicine to assess various types of diseases. It has also provided a clear perspective on laser therapy. Some cancers, gastrointestinal diseases, neurodegenerative disorders, and many diseases are evaluated via PPI analysis. ${ }^{15-18}$ In the present study, 6 proteins which are dysregulated in response to LLLT in plasma are assessed via PPI network analysis to find possible critical proteins (among the query proteins and also the 100 first neighbors) which are involved in LLLT effects on the body.

\section{Materials and Methods}

To assess the effect of LLLT on the body, 6 proteins which are identified as dysregulated proteins after laser application to rats were extracted from the published data by Kilik et al. ${ }^{19}$ Based on the explained methods of that paper, 2 groups of 5-month-old rats were assigned as control and irradiated groups. The exposed group was irradiated for 9 days with a total daily dose of 60.3 $\mathrm{J} / \mathrm{cm}^{2}$ with 134 irradiation time. Plasma proteins of the 2 groups were made via two-dimensional electrophoresis and 6 differentiated spots including HP, HPX, SERPINA1, FGG, FETUB, and AHSG were identified by the mass spectrometer MALDI TOF/TOF UltrafleXtreme. The details of the methods are explained in the original study. ${ }^{19}$

The 6 query proteins interacted as an interactome unit by Cytoscape software v3.7.2 via the protein query of the STRING database. ${ }^{20}$ To find more informative data, 100 first neighbors were added to the 6 query proteins and a network was constructed. The network was analyzed by the network analyzer application of Cytoscape and the central nodes based on centrality parameters were determined. Ten percent of the top nodes based on degree value, betweenness centrality, closeness centrality (CC), and stress were identified as central nodes. The central nodes were connected by the STRING database (https:// string-db.org/cgi/network?taskId=bik4WQAVFNg9\&s essionId=b4ILsdOYrIb2) and also the description of the central proteins was extracted from STRING.

\section{Results}

The 6 query proteins interacted (see Figure 1) to conform to an interactome unit. All of the proteins were connected to each other. To access more information, 100 neighbors were added to the query proteins and the constructed network (Figure 2) was analyzed.

Among the 106 nodes, 10 individuals (about 10\%) which were characterized by top amounts of degree were identified as hub nodes. As it is shown in Table 1, the determined hubs are categorized by the highest values of betweenness centrality, CC, and stress. Therefore, the hubs were assigned as central proteins. Connections between the central nodes are presented in Figure 3. All

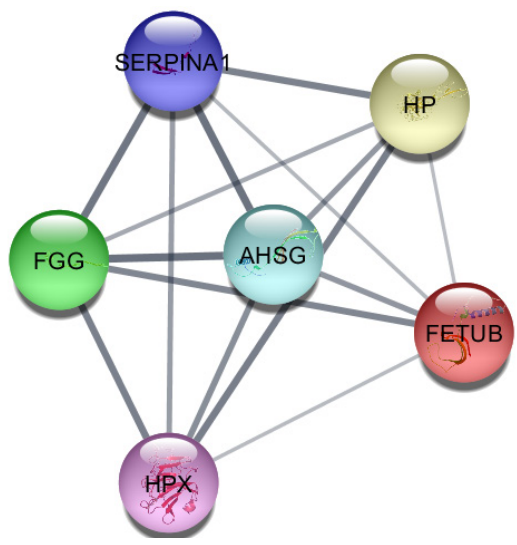

Figure 1. The Interactome Unit Including the 6 Query Proteins. Each node is linked to the other proteins.

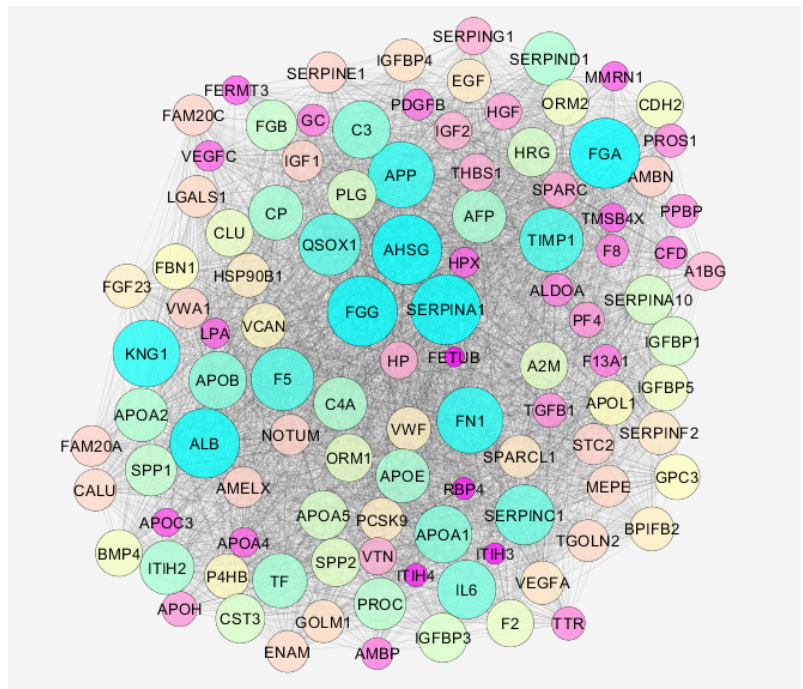

Figure 2. The Network Including 6 Query Proteins and the Related 100 First Neighbors. The nodes are layout based on degree value. Color from pink to blue refers to the degree value increment. The bigger size of the node corresponds to larger degree value. The 6 query proteins (AHSG, SERPINA1, HP, HPX, FETUB, AND FGG) are presented in the center of the network.

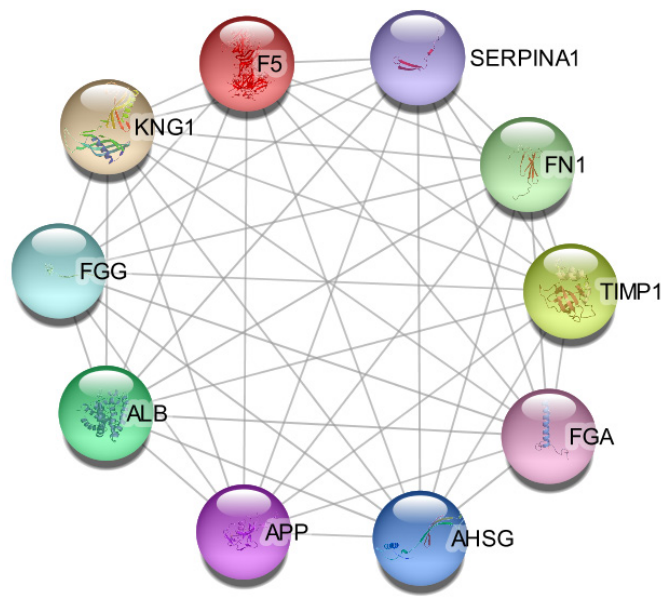

Figure 3. The Sub-network Including the 10 Central Proteins. 
of the central nodes are connected to each other with full capacity. The description of the central proteins (see Table 2) is extracted from STRING (https://string-db.org).

\section{Discussion}

Many attempts are required to achieve an understanding of the mechanism of LLLT and its side effect on the human body. ${ }^{21,22}$ Proteomics is represented as a suitable ability in exploring the molecular mechanism of different cases of diseases or therapeutic methods. ${ }^{23}$ In the original study, 6 proteins which were dysregulated after LLLT in the serum of rats were introduced. Network analysis provided the possibility to rate the biological impact of this set of proteins besides the introduction of new proteins that are related to LLLT. The 6 proteins were categorized into 2 groups including the first group (AHSG, FGG, and SERPINA1) and the second group (TP, TPX, and FETUB). The elements of the first group were marked with degree values of 105 while degree values of HP, HPX, and FETUB

Table 1. The 10 Central Proteins

\begin{tabular}{lcccc}
\hline Gene name & $\mathbf{K}$ & $\mathbf{B C}$ & $\mathbf{C C}$ & Stress \\
\hline AHSG & 105 & 0.018 & 1.000 & 4350 \\
FGA & 105 & 0.018 & 1.000 & 4350 \\
FGG & 105 & 0.018 & 1.000 & 4350 \\
SERPINA1 & 105 & 0.018 & 1.000 & 4350 \\
ALB & 104 & 0.018 & 0.991 & 4200 \\
KNG1 & 100 & 0.015 & 0.955 & 3664 \\
FN1 & 99 & 0.015 & 0.946 & 3576 \\
APP & 98 & 0.014 & 0.938 & 3434 \\
TIMP1 & 94 & 0.012 & 0.905 & 2946 \\
F5 & 93 & 0.012 & 0.897 & 2844 \\
\hline
\end{tabular}

$\overline{\text { Note. K, BC, and CC refer to degree, betweenness centrality, and closeness }}$ centrality respectively. were 53,43 , and 30 respectively.

The analysis led to introducing 7 neighbor proteins including FGA, ALB, KNG1, FN1, APP, TIMP1, and F5 as high impact proteins in response to LLLT action. As it is tabulated in Table 2, the following terms are attributed to the 10 central proteins: Promotion of endocytosis, involvement in the mineral phase of the bone, the role in wound repair, platelet aggregation, body hemostasis, blood clots, inhibition of trypsin, chymotrypsin and plasminogen activation, decrease of coagulation time, proteolytic activity against insulin and plasmin, binding capacity for water, $\mathrm{Ca}(2+), \mathrm{Na}(+), \mathrm{K}(+)$, fatty acids, hormones, bilirubin and drugs, regulation of the colloidal osmotic pressure of blood, transport of zinc in plasma, smooth muscle contraction, induction of hypotension, natriuresis and diuresis, degeneration of both neuronal cell bodies and axons, regulation of cell differentiation, migration and cell death and activation, cellular signaling cascades, integrin signaling, erythropoiesis, decrease in the blood glucose level, and mediation of inflammation. It seems the terms are related mainly to body hemostasis.

The investigation revealed that the saliva albumin level changed significantly after LLLT. In this report, it was found that the level of secretory IgA and albumin after radiation (diode $670 \mathrm{~nm}$ and Helium-Neon 632.8 $\mathrm{nm}$ ) were altered in the saliva of the irradiated human. ${ }^{24}$ Huang et al evaluated proteomics of acute radiation syndrome of the murine gastrointestinal tract. Based on the finding of this research, KNG1 is dysregulated after radiation. It was expressed that MAPK1, AHSG, C3, MAP2k3, APOE, KNG1, S100A8, and CD36 that were associated with inflammation were dysregulated after the radiation process. ${ }^{25} \mathrm{Kim}$ et al published data about the effects of low-intensity laser therapy on rats. They reported that immunoreactivity of TIMP1 was inhibited by low-intensity laser therapy. ${ }^{26}$ These findings indicate

Table 2. Description of 10 Central Proteins, Extracted From STRING (https://string-db.org)

\begin{tabular}{|c|c|}
\hline Protein & Description \\
\hline AHSG & It promotes endocytosis, possesses opsonic properties and influences the mineral phase of the bone. It shows affinity for calcium and barium ions \\
\hline FGA & $\begin{array}{l}\text { It functions during the early stages of wound repair to stabilize the lesion and guide cell migration during re-epithelialization. It was originally } \\
\text { thought to be essential for platelet aggregation. }\end{array}$ \\
\hline FGG & $\begin{array}{l}\text { It has a major function in hemostasis as one of the primary components of blood clots. In addition, it functions during the early stages of wound } \\
\text { repair to stabilize the lesion and guide cell migration during re-epithelialization. It was originally thought to be essential for platelet aggregation. }\end{array}$ \\
\hline SERPINA1 & $\begin{array}{l}\text { It irreversibly inhibits trypsin, chymotrypsin and plasminogen activator. The aberrant form inhibits insulin-induced NO synthesis in platelets, } \\
\text { decreases coagulation time and has proteolytic activity against insulin and plasmin }\end{array}$ \\
\hline ALB & $\begin{array}{l}\text { It has a good binding capacity for water, } \mathrm{Ca}(2+), \mathrm{Na}(+), \mathrm{K}(+) \text {, fatty acids, hormones, bilirubin and drugs. Its main function is the regulation of the } \\
\text { colloidal osmotic pressure of blood. The major zinc transporter in plasma typically binds about } 80 \% \text { of all plasma zinc. }\end{array}$ \\
\hline KNG1 & $\begin{array}{l}\text { It plays an important role in blood coagulation, inhibits the thrombin- and plasmin-induced aggregation of thrombocytes, influences smooth } \\
\text { muscle contraction, induces hypotension, natriuresis and diuresis, decreases the blood glucose level, and is a mediator of inflammation }\end{array}$ \\
\hline FN1 & Endogenous ligands \\
\hline APP & It triggers caspase activation and degeneration of both neuronal cell bodies (via caspase-3) and axons (via caspase-6). \\
\hline TIMP1 & $\begin{array}{l}\text { It functions as a growth factor that regulates cell differentiation, migration and cell death and activates cellular signaling cascades via CD63 and } \\
\text { ITGB1. It plays a role in integrin signaling. It mediates erythropoiesis in vitro. }\end{array}$ \\
\hline F5 & It is the central regulator of hemostasis. \\
\hline
\end{tabular}


that the neighbor proteins are involved in the alteration after radiation application. In summary, 2 parameters were considered to find the effective proteins in response to radiation in the body; first, the direct involvement of the protein in response to radiation, and second, the important role of the explored proteins. Our analysis showed that $50 \%$ of the introduced proteins including FGG, AHSG, and SERPINA1 are the critical proteins and the others (HP, HPX, and FETUB) have a mild role in body response to radiation. Furthermore, in the present study, 7 neighbor proteins were introduced as effective proteins to respond to radiation; however, they were not presented in the original research.

\section{Conclusion}

FGG, AHSG, SERPINA1, TIMP1, KNG1, F5, APP, FGA, $\mathrm{FN} 1$, and ALB were introduced as the critical proteins that are involved in response to LLLT. Significant roles of the determined proteins are recognized as the regulation of body hemostasis and wound repair. The findings support the application of LLLT in medicine.

\section{Ethical Considerations \\ Not applicable.}

\section{Conflict of Interests}

There is no conflict of interest.

\section{Acknowledgments}

This project was supported by Shahid Beheshti University of Medical Sciences.

\section{References}

1. Posten W, Wrone DA, Dover JS, Arndt KA, Silapunt S, Alam M. Low-level laser therapy for wound healing: mechanism and efficacy. Dermatol surg. 2005;31(3):334-40. doi: $10.1111 /$ j.1524-4725.2005.31086

2. Sun G, Tunér J. Low-level laser therapy in dentistry. Dent Clin North Am. 2004;48(4):1061-76, viii. doi: 10.1016/j. cden.2004.05.004.

3. Hashmi JT, Huang YY, Osmani BZ, Sharma SK, Naeser MA, Hamblin MR. Role of low-level laser therapy in neurorehabilitation. $\quad P M \quad$ R. 2010;2:S292-S305. doi: 10.1016/j.pmrj.2010.10.013.

4. Smith KC. The photobiological basis of low level laser radiation therapy. Laser Therapy. 1991;3(1):19-24. doi: 10.5978/islsm.91-OR-03.

5. Mussttaf RA, Jenkins DF, Jha AN. Assessing the impact of low level laser therapy (LLLT) on biological systems: a review. Int J Radiat Biol. 2019;95(2):120-43. doi: 10.1080/09553002.2019.1524944.

6. Hanash, S. Disease proteomics. Nature. 2003;422:226-32. doi: 10.1038/nature01514.

7. Shahrokh S, Razzaghi Z, Mansouri V, Ahmadi N. The impact of proteomic investigations on the development and improvement of skin laser therapy: a review article. J Lasers Med Sci. 2019;10(Suppl 1):S90-S95. doi: 10.15171/ jlms.2019.S16.
8. Van JA, Scholey JW, Konvalinka A. Insights into diabetic kidney disease using urinary proteomics and bioinformatics. J Am Soc Nephrol. 2017;28(4):1050-61. doi: 10.1681/ASN.2016091018.

9. Dong W, Qiu C, Gong D, Jiang X, Liu W, Liu W, et al. Proteomics and bioinformatics approaches for the identification of plasma biomarkers to detect Parkinson's disease. Exp Ther Med. 2019;18(4):2833-42. doi: 10.3892/ etm.2019.7888.

10. Werner T. Bioinformatics applications for pathway analysis of microarray data. Curr Opin Biotechnol. 2008;19(1):50-4. doi: 10.1016/j.copbio.2007.11.005.

11. Laukens K, Naulaerts S, Berghe WV. Bioinformatics approaches for the functional interpretation of protein lists: from ontology term enrichment to network analysis. Proteomics. 2015;15(5-6):981-96. doi: 10.1002/ pmic.201400296.

12. de Oliveira Poswar F, Farias LC, de Carvalho Fraga CA, Bambirra W Jr, Brito-Júnior M, Sousa-Neto MD, et al. Bioinformatics, interaction network analysis, and neural networks to characterize gene expression of radicular cyst and periapical granuloma. J endod. 2015;41(6):877-83. doi: 10.1016/j.joen.2015.02.004.

13. Teschendorff AE, Banerji CR, Severini S, Kuehn R, Sollich $P$. Increased signaling entropy in cancer requires the scalefree property of protein interaction networks. Sci Rep. 2015;5:9646. doi: 10.1038/srep09646.

14. He X, Zhang J. Why do hubs tend to be essential in protein networks?. PLoS Genet. 2006;2(6): e88. doi: 10.1371/ journal.pgen.0020088.

15. Rezaei-Tavirani M, Rezaei Tavirani M, Zamanian Azodi M, Moravvej Farshi H, Razzaghi M. Evaluation of skin response after erbium:yttrium- aluminum-garnet laser irradiation: a network analysis approach. J Lasers Med Sci. 2019;10(3):194-199. doi: 10.15171/jlms.2019.31.

16. Rostami-Nejad M, Rezaei-Tavirani M, Zadeh-Esmaeel MM, RezaeiTavirani S, Akbari Z, Esmaeili S, Okhovatian F. Assessment of cytokine-mediated signaling pathway dysregulation in arm skin after CO2 laser therapy. J Lasers Med Sci. 2019;10(4):257-63. doi: 10.15171/jlms.2019.42.

17. Mansouri V, Rezaei-Tavirani M, Zadeh-Esmaeel MM, Rezaei-Tavirani S, Razzaghi M, Okhovatian F, et al. Analysis of laser therapy effects on squamous cell carcinoma patients: a system biology study. J Lasers Med Sci. 2019;10(Suppl 1):S1-S6. doi: 10.15171/jlms.2019.S1.

18. Zamanian-Azodi M, Rezaei-Tavirani M, Rahmati-Rad S, Hasanzadeh H, Rezaei Tavirani M, Seyyedi SS. Proteinprotein interaction network could reveal the relationship between the breast and colon cancer. Gastroenterol Hepatol Bed Bench. 2015;8(3):215-24.

19. Kilik R, Bober P, Ropovik I, Beňačka R, Genči J, Nečas A, et al. Proteomic Analysis of Plasma Proteins after LowLevel Laser Therapy in Rats. Physiolog Res. 2019;68(Suppl 4):S399-S404

20. Wu J, Ruan D-D, Fu T-F, Yuan J. A network pharmacology study of reduning injection for the treatment of coronavirus disease-19. World J Tradit Chin Med. 2020;6(2):180. doi: 10.4103/wjtcm.wjtcm_19_20.

21. Hawkins D, Houreld N, Abrahamse H. Low level laser therapy (LLLT) as an effective therapeutic modality for delayed wound healing. Ann N Y Acad Sci. 
2005;1056(1):486-93. doi: 10.1196/annals.1352.040

22. Suzuki SS, Garcez AS, Reese PO, Suzuki H, Ribeiro MS, Moon W. Effects of corticopuncture (CP) and low-level laser therapy (LLLT) on the rate of tooth movement and root resorption in rats using micro-CT evaluation. Lasers Med Sci. 2018;33(4):811-21. doi: 10.1007/s10103-0172421-5.

23. Butterfield DA, Boyd-Kimball D, Castegna A. Proteomics in Alzheimer's disease: insights into potential mechanisms of neurodegeneration. J Neurochem. 2003;86(6):1313-27. doi: 10.1046/j.1471-4159.2003.01948.x.

24. KUČCEROVÁ H, DOSTÁlOVÁ T, HIMMLOVÁ L, BÁRTOVÁ J, MAZÁNEK J. Low-level laser therapy after molar extraction. J Clin Laser Med Surg. 2000;18(6):30915. doi: $10.1089 / \mathrm{clm} .2000 .18 .309$.

25. Huang W, Yu J, Jones JW, Carter CL, Pierzchalski K, Tudor G, Booth C, MacVittie TJ, Kane MA. Proteomic evaluation of the acute radiation syndrome of the gastrointestinal tract in a murine total-body irradiation model. Health Phys. 2019;116(4):516-28. doi: 10.1097/HP.0000000000000951.

26. Kim S-J, Kang Y-G, Park J-H, Kim E-C, Park Y-G. Effects of low-intensity laser therapy on periodontal tissue remodeling during relapse and retention of orthodontically moved teeth. Lasers Med Sci. 2013;28(1):325-33. doi: 10.1007/s10103-012-1146-8. 